\title{
PPAR $\gamma$-induced cardiolipotoxicity in mice is ameliorated by PPAR $\alpha$ deficiency despite increases in fatty acid oxidation
}

\author{
Ni-Huiping Son, ${ }^{1}$ Shuiqing Yu, ${ }^{1}$ Joseph Tuinei, ${ }^{2}$ Kotaro Arai, ${ }^{3}$ Hiroko Hamai, ${ }^{4}$ Shunichi Homma, ${ }^{3}$ \\ Gerald I. Shulman, ${ }^{5}$ E. Dale Abel, ${ }^{2}$ and Ira J. Goldberg ${ }^{1}$ \\ 'Division of Preventive Medicine and Nutrition, Columbia University, New York, New York, USA. ${ }^{2}$ Division of Endocrinology Metabolism and Diabetes and \\ Program in Molecular Medicine, University of Utah, Salt Lake City, Utah, USA. ${ }^{3}$ Division of Cardiology, Department of Medicine, and \\ ${ }^{4}$ Department of Pathology and Cell Biology, Columbia University, New York, New York, USA. ${ }^{5}$ Department of Medicine, \\ Yale University School of Medicine, New Haven, Connecticut, USA.
}

\begin{abstract}
Excess lipid accumulation in the heart is associated with decreased cardiac function in humans and in animal models. The reasons are unclear, but this is generally believed to result from either toxic effects of intracellular lipids or excessive fatty acid oxidation (FAO). PPAR $\gamma$ expression is increased in the hearts of humans with metabolic syndrome, and use of PPAR $\gamma$ agonists is associated with heart failure. Here, mice with dilated cardiomyopathy due to cardiomyocyte PPAR $\gamma$ overexpression were crossed with PPAR $\alpha$-deficient mice. Surprisingly, this cross led to enhanced expression of several PPAR-regulated genes that mediate fatty acid (FA) uptake/oxidation and triacylglycerol (TAG) synthesis. Although FA oxidation and TAG droplet size were increased, heart function was preserved and survival improved. There was no marked decrease in cardiac levels of triglyceride or the potentially toxic lipids diacylglycerol (DAG) and ceramide. However, long-chain FA coenzyme A (LCCoA) levels were increased, and acylcarnitine content was decreased. Activation of PKC $\alpha$ and PKC $\delta$, apoptosis, ROS levels, and evidence of endoplasmic reticulum stress were also reduced. Thus, partitioning of lipid to storage and oxidation can reverse cardiolipotoxicity despite increased DAG and ceramide levels, suggesting a role for other toxic intermediates such as acylcarnitines in the toxic effects of lipid accumulation in the heart.
\end{abstract}

\section{Introduction}

With the increase in the prevalence of obesity and type 2 diabetes, a series of disorders associated with ectopic deposition of fat have become more common and are termed lipotoxic diseases (1). Although the clinical presentations are in diverse tissues - leading to nonalcoholic fatty liver disease, muscle insulin resistance, and cardiac dysfunction - it is likely that they have common or overlapping pathophysiology. The heart is the most energy-demanding tissue of the body and utilizes fatty acids (FAs) as its major source of substrate for ATP generation (2). Nonetheless, excess FA oxidation (FAO) has been implicated as a cause of cardiac dysfunction in obesity and diabetes $(3,4)$. In humans, greater stores of cardiac lipid in obesity $(5,6)$ and type 2 diabetes (7) are correlated with reduced heart function.

Several genetically modified animals were created to have altered cardiac lipid content and determine how this affects heart function exclusive of systemic metabolic changes. Overexpression of fatty acyl-CoA synthetase (8), a cardiomyocyte cell surface-anchored form of lipoprotein lipase (9), or FA transport protein (10) augments heart lipid content and leads to cardiomyopathy. Overexpression of PPAR $\alpha$ (4) and PPAR $\gamma$ (11) using the $\alpha$-myosin heavy chain (MHC) promoter led to cardiac lipid accumulation and cardiomyopathy; the transgenes are designated MHC-Ppara and MHC-Pparg. Although this was associated with increased expression of genes controlling FAO, these hearts accumulated more triacylglycerol (TAG). Thus, the increase in lipid uptake likely outstrips the greater FAO. The dilated cardiomyopathy in these animals, especially the MHC-Ppara transgenic mice, has been com-

Conflict of interest: The authors have declared that no conflict of interest exists. Citation for this article: J Clin Invest. 2010;120(10):3443-3454. doi:10.1172/JCI40905. pared with that occurring with diabetes (4). The pathophysiology of the cardiac dysfunction with PPAR $\alpha$ and PPAR $\gamma$ overexpression is unclear but has been hypothesized to result from excess FAO or accumulation of toxic intracellular lipids (12).

PPAR $\gamma$ agonists cause heart failure in humans. One reason for this may be greater accumulation of salt and water (13). However, potent PPAR $\gamma$ agonists cause cardiomegaly in rodents (14). The ratio of PPAR $\gamma /$ PPAR $\alpha$ expression in human hearts is much greater than in mice (15). Moreover, a recent report showed that PPAR $\gamma$ expression is markedly increased in ventricular muscle from subjects with metabolic syndrome (16). Thus, aside from serving as a model to understand the toxic effects of lipid in the heart, MHCPparg transgenic mice are likely to mimic pathological processes that occur with PPAR $\gamma$ agonist treatment of patients who have a predisposition to developing lipotoxic cardiomyopathy.

Greater intracellular toxic lipid content might lead to heart dysfunction associated with ceramide-induced apoptosis (17), increased ROS formation, mitochondrial dysfunction $(18,19)$, and/or ER stress (20). We hypothesized that deletion of PPAR $\alpha$ in the heart would reduce the expression of FAO genes and FAO in the MHC-Pparg hearts and would ameliorate cardiac dysfunction. To study this, we crossed MHC-Pparg mice with PPAR $\alpha$-knockout $\left(\mathrm{Ppara}^{-1}\right)$ mice. Surprisingly MHC-Pparg/Ppara/- mice had greater expression of PPAR downstream genes, including those responsible for FAO, and greater FAO. These mice, however, had improved cardiac function and survival. Many potentially toxic pathways were simultaneously improved in the MHC-Pparg/Ppara-/- mice; therefore, no single cause of the improvement can be defined. Nonetheless, our studies show that lipotoxicity is ameliorated despite greater FAO and no reduction in TAG storage. 
Table 1

PPAR $\alpha$ deficiency increased plasma FA and decreased glucose levels in MHC-Pparg mice

\begin{tabular}{lcccc}
\hline & $\begin{array}{c}\text { Control } \\
(\boldsymbol{n}=\mathbf{1 1})\end{array}$ & $\begin{array}{c}\text { MHC-Pparg } \\
(\boldsymbol{n}=\mathbf{1 3})\end{array}$ & $\begin{array}{c}\text { Ppara- } \\
(\boldsymbol{n}=\mathbf{1 4})\end{array}$ & $\begin{array}{c}\text { MHC-Pparg/Ppara-/ } \\
(\boldsymbol{n}=\mathbf{1 0})\end{array}$ \\
& $1.20 \pm 0.08$ & $1.21 \pm 0.26$ & $1.52 \pm 0.17^{\mathrm{A}, \mathrm{B}}$ & $1.51 \pm 0.26^{\mathrm{A}, \mathrm{B}}$ \\
FFA (mol/l) & $70.2 \pm 14.1$ & $67.6 \pm 16.5$ & $62.4 \pm 11.3$ & $54.7 \pm 8.5^{\mathrm{A}, \mathrm{B}}$ \\
TG $(\mathrm{mg} / \mathrm{dl})$ & $107.6 \pm 21.1$ & $110.7 \pm 18.8$ & $119.3 \pm 14.8$ & $106.9 \pm 17.3$ \\
TC $(\mathrm{mg} / \mathrm{dl})$ & $69.6 \pm 13.1$ & $60.4 \pm 8.5$ & $42.1 \pm 4.0^{\mathrm{A}, \mathrm{B}}$ & $41.2 \pm 8.8^{\mathrm{A}, \mathrm{B}}$ \\
Glucose $(\mathrm{mg} / \mathrm{dl})$ & $4.56 \pm 0.15$ & $5.59 \pm 0.74 \mathrm{~A}$ & $4.74 \pm 0.58$ & $5.71 \pm 0.58^{\mathrm{A}}$ \\
Heart/body wt ratio $(\mathrm{mg} / \mathrm{g})$ & & & & \\
\hline
\end{tabular}

Ten- to 12 -week-old male mice fasted for 18 hours. Data are shown as mean $\pm \mathrm{SD}$. ${ }^{A} P<0.05$ vs. control mice. ${ }^{\mathrm{B} P}<0.05$ versus $\mathrm{MHC}-\mathrm{Pparg}$ mice.

\section{Results}

Generation of MHC-Pparg/Ppara-/- mice. To study the effects of PPAR $\alpha$ deficiency in the PPAR $\gamma$-mediated cardiomyopathy, we crossed the high-expressing MHC-Pparg mouse line (15) twice into the $\mathrm{Ppara}^{-/}$background, which resulted in MHC-Pparg/Ppara-/ offspring. Overexpression of PPAR $\gamma$ and the absence of PPAR $\alpha$ in the heart were confirmed in each genotype at the protein level (Supplemental Figure 1; supplemental material available online with this article; doi:10.1172/JCI40905DS1).

Circulating plasma metabolites. PPAR $\alpha$ deficiency is associated with increased circulating FFAs (Table 1). In MHC-Pparg mice, FFA concentrations were similar to those in controls. In MHC-Pparg/Ppara-/mice, FFAs were equivalent to those in $\mathrm{Ppara}^{-/-}$mice. TAG levels have been reported to be variable in the Pparal- mice $(21,22)$. In our mice, they were not significantly reduced compared with those in control or MHC-Pparg mice, but this reduction reached significance when the MHCPparg/Ppara-/- mice were compared with either control or MHC-Pparg animals. There were no statistical differences in plasma cholesterol among these mice. PPAR $\alpha$ deficiency is associated with decreased circulating glucose. In MHC-Pparg mice, glucose concentrations were similar to those in controls; and in MHC-Pparg/Ppara ${ }^{-/-}$mice, glucose concentrations were equivalent to those in $\mathrm{Ppara}^{-/}$mice.

PPAR $\alpha$ deficiency ameliorates heart dysfunction and increases survival of MHC-Pparg mice. Heart weights of PPAR $\gamma$ mice were increased, and this increase was not reduced in MHC-Pparg/Ppara ${ }^{-/}$mice (Figure 1A). However, echocardiography showed improved heart function in 3-month-old male MHC-Pparg/Ppara-/- compared with MHC-Pparg mice. As expected, MHC-Pparg mice exhibited reduced fractional shortening (FS) and increased systolic left ventricular dimensions (LVDs) compared with wild-type controls (Figure 1, B-D). In stark contrast, LV function of the MHC-Pparg/Ppara-mice was improved and was not different from that of the wildtype control mice. Consistent with the echocardiography data,
A

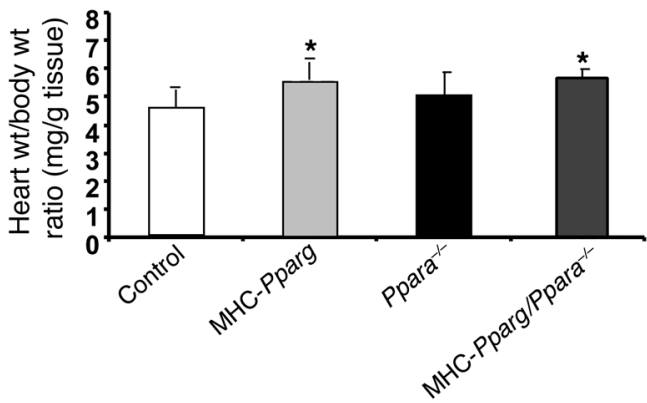

B

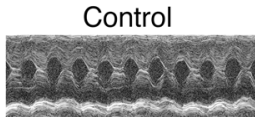

Ppara $^{-1-}$
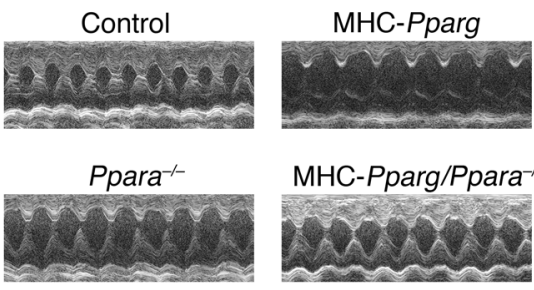

MHC-Pparg/Ppara-1-

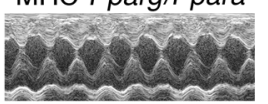

C

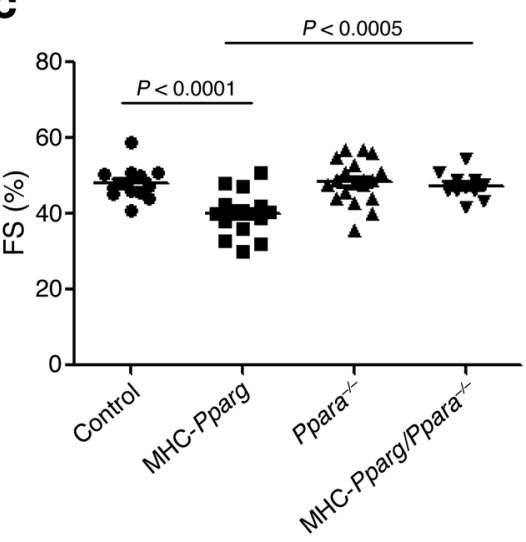

D

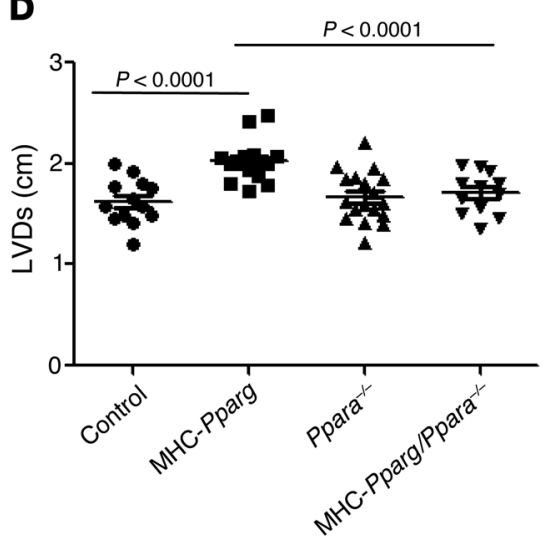

E

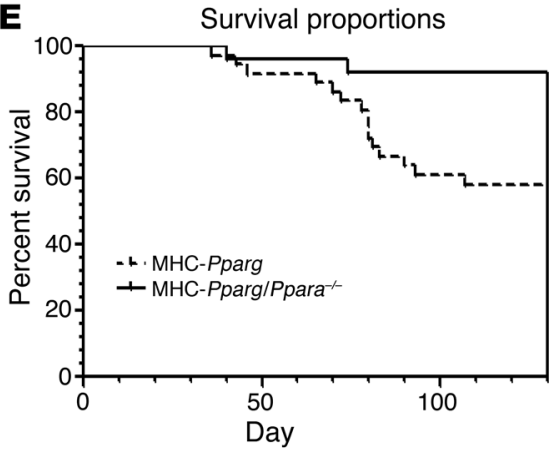

Figure 1

PPAR $\alpha$ deficiency improved heart function and increased survival rates in MHC-Pparg mice. (A) Heart weight to body weight ratio in mice $(n=11-18)$. (B) Representative M-mode echocardiographic images of LVD in MHC-Pparg and MHC-Pparg/Ppara ${ }^{-/-}$mice. (C and D) Echocardiography showed increased FS and reduced LVDs in MHC-Pparg/Ppara ${ }^{-/}$mice $(n=11-18)$. (E) Survival was increased in MHC-Pparg/Ppara ${ }^{-/-}$ mice. Data are shown as mean $\pm \mathrm{SD} .{ }^{*} P<0.05$ versus normal controls. 
A

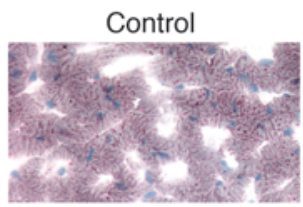

B

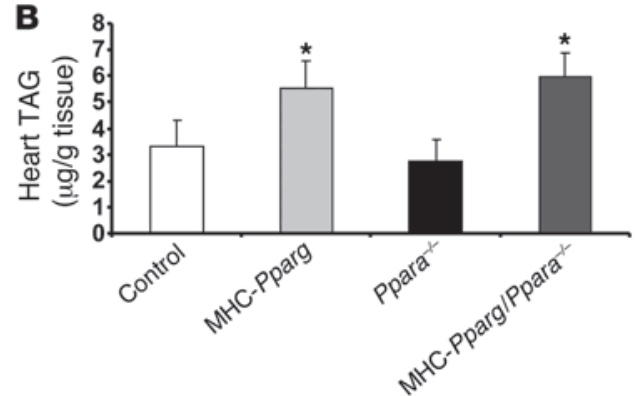

MHC-Pparg
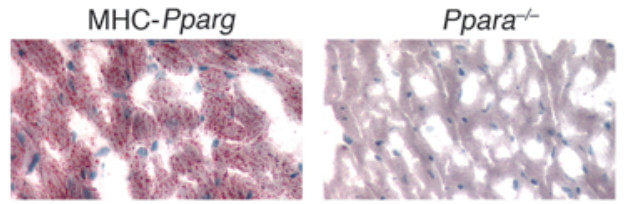

MHC-Pparg/Ppara ${ }^{-}$

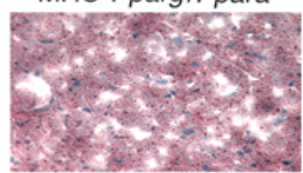

C

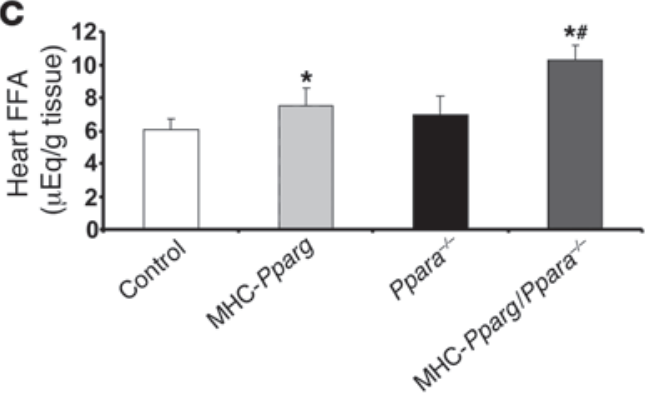

Figure 2

Accumulation of intracellular lipid in the heart of MHC-Pparg and MHC-Pparg/Ppara-/- mice. (A) Oil red O staining showed an abundance of neutral lipid droplets randomly scattered throughout the cytoplasm of cardiomyocytes in both MHC-Pparg and MHC-Pparg/Ppara ${ }^{-/-}$mice after overnight fasting (original magnification, $\times 200$ ). (B) Heart TAG and (C) FFA content were significantly increased in both MHC-Pparg and MHC-Pparg/Ppara ${ }^{--}$mice compared with control mice $(n=7)$. Data are shown as mean $\pm \mathrm{SD}$. ${ }^{*} P<0.05$ versus littermate controls; ${ }^{*} P<0.05$ versus MHC-Pparg mice.

the survival rate increased from 58\% to $92 \%$ at 130 days in MHCPparg/Ppara ${ }^{-1}$ mice $(P<0.05$, Figure 1E).

$P P A R \alpha$ deficiency does not reduce cardiac lipid content. The finding of improved cardiac function with persistently increased cardiac size was surprising. To assess whether PPAR $\alpha$ deficiency altered heart lipid accumulation, we stained heart tissues with oil red $\mathrm{O}$. As shown in Figure 2A, MHC-Pparg hearts had more lipid than wild-type, whereas $\mathrm{Ppara}^{-/}$hearts had less neutral lipid. Despite the improved cardiac function, loss of PPAR $\alpha$ did not reduce lipid accumulation in the hearts of MHC-Pparg mice. Both MHC-Pparg and MHC-Pparg/Ppara-- mice had copious amounts of stained lipids. Heart tissue TAG data was consistent with the oil red O staining pattern, demonstrating significantly higher TAG levels in both MHC-Pparg and MHC-Pparg/Ppara-/- mice (Figure 2B). Heart tissue FFA levels were significantly increased in MHC-Pparg/Ppara-/- mice compared with MHC-Pparg mice (10.03 \pm 0.86 vs. $7.51 \pm 1.01 \mathrm{~mol} / \mathrm{l}$, $P<0.01$ ) (Figure 2C); FFA levels in $\mathrm{Ppara}^{-/-}$and control hearts were similar. MHC-Pparg hearts had a significant increase in palmitic acid (C16:0) and decrease in docosahexaenoic acid (C22:6n3) (DHA) and docosapentaenoic acid (C22:5n6) (DPA) concentrations compared with control, but MHC-Pparg and MHC-Pparg/Ppara-/hearts had a similar FA distribution (Supplemental Figure 2). These data suggested that the improved function of MHC-Pparg/Ppara-hearts was not due to reduction in palmitate.

Ceramide levels were increased in MHC-Pparg mice compared with control mice (Figure $3 \mathrm{~A}$ ) but were not altered by crossing the MHC-Pparg transgene onto the Ppara/- background. However, C24:1 and C24 ceramides, which have been shown to be more toxic $(23,24)$, were slightly but significantly decreased in MHC-Pparg/Ppara-/- mice (Figure 3B). DAG, measured using the DAG kinase method, was also increased, but neither the level nor the distribution correlated with the improved cardiac function (Supplemental Figure 3A).

Long-chain FA coenzyme A (LCCoA) and acylcarnitine are intermediates in lipid oxidation. PPAR $\alpha$ deficiency significantly increased total intracellular LCCoA content in MHC-Pparg hearts $(86.5 \pm 10.4 \mathrm{nmol} / \mathrm{g}$ vs. $61.9 \pm 9.7 \mathrm{nmol} / \mathrm{g} ; P<0.01)$ (Figure $3 \mathrm{C}$ and Supplemental Figure 3B), consistent with reduced FAO. LCCoAs are converted to acylcarnitines prior to mitochondrial FA $\beta$-oxidation; LCCoA can also be utilized for peroxisome $\beta$-oxidation and TAG synthesis $(25,26)$. Despite increased LCCoA content, acylcarnitine content was reduced in MHC-Pparg/Ppara ${ }^{-/}$compared with MHC-Pparg mice $(1,189.7 \pm 163.5 \mathrm{nmol} / \mathrm{g}$ vs. $1,611.7 \pm 98.6 \mathrm{nmol} / \mathrm{g}$; $P<0.01$ ) (Figure 3D), especially associated with a reduction in medium- and long-chain acylcarnitines (Supplemental Figure 3C). Because acylcarnitine is a toxic catabolite (27-29), reduction of acylcarnitine content and increased TAG storage might have, at least partially, contributed to amelioration of cardiac dysfunction in MHC-Pparg/Ppara ${ }^{-/}$mice.

Lipid droplet morphology is altered in cardiomyocytes from MHC-Pparg/ $\mathrm{Ppara}^{-/}$mice. Electron microscopy showed distorted mitochondrial contours and more lipid droplets within the sarcoplasm of cardiomyocytes in the MHC-Pparg and MHC-Pparg/Ppara-/- mice. MHC-Pparg/P $\mathrm{para}^{-/-}$hearts showed a striking redistribution of lipid storage into large droplets (Figure 4A). In some areas of MHC-Pparg/Ppara ${ }^{-/}$hearts, lipid droplets were surrounded by mitochondria (Figure 4A). In addition, as shown at higher power, disrupted cristae were seen in MHC-Pparg but not in MHC-Pparg/ $P_{p a r a-1-}$ hearts (Figure 4B). Lipid droplet size was significantly increased in MHC-Pparg/Ppara-/- mice compared with MHCPparg mice $(1.93 \pm 0.81$ vs. $0.77 \pm 0.32 \mu \mathrm{m}, P<0.001)$ (Figure 4C). MHC-Pparg mice had greater cardiac uptake of VLDL-TAG than wild-type or $\mathrm{Ppara}^{-/-}$mice (Figure 4D), and this was not reduced in MHC-Pparg/Ppara-/- mice. Cardiac 2-deoxyglucose uptake was increased in MHC-Pparg and Ppara-/- mice but was not further increased in MHC-Pparg/Ppara ${ }^{-/}$mice (Figure 4E).

MHC-Pparg/Ppara ${ }^{--}$mice have increased cardiac lipid oxidation and DNA copy number. To further investigate the metabolism of these hearts, we determined palmitate oxidation and myocardial oxygen consumption $\left(\mathrm{MVO}_{2}\right)$ in isolated working hearts (30). Rates of pal- 
A
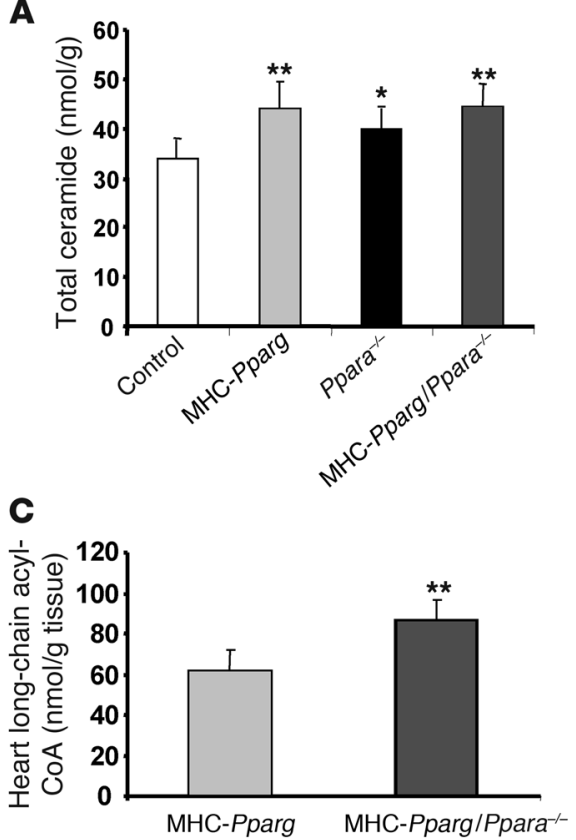
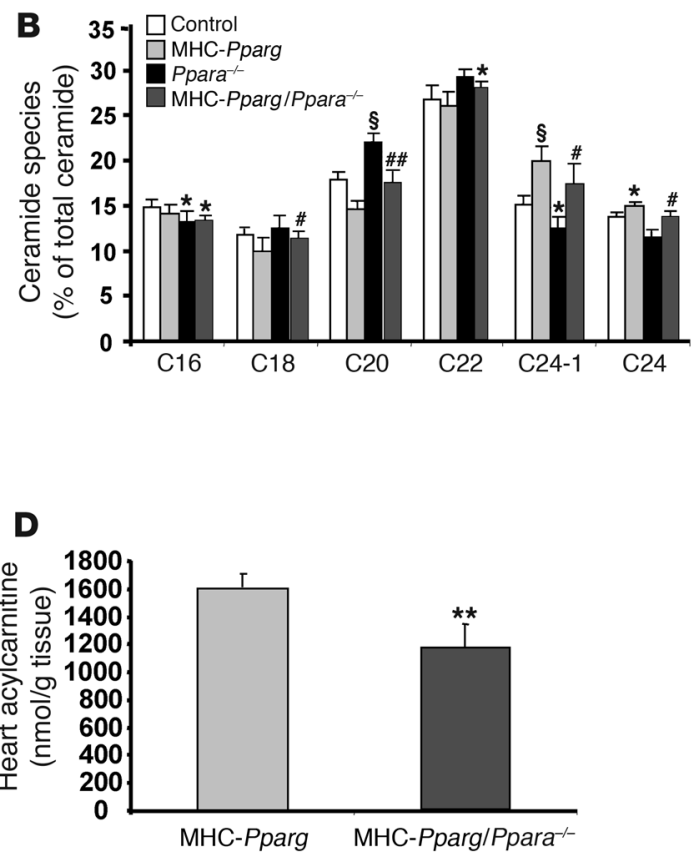

Figure 3

Total ceramide, long-chain acyl-CoA, and acylcarnitine content in hearts of MHC-Pparg and MHC-Pparg/Ppara-/- mice. (A) Total ceramide and (B) individual ceramide species. Ceramide species data represent the content of each FA as a percentage of total ceramide and are shown as mean $\pm \mathrm{SD}\left(n=6-7\right.$ per group). (C) Total long-chain acyl-CoA and (D) acetylcarnitine content. Data are shown as mean \pm SD. ${ }^{*}<0.01$, ${ }^{\star \star} P<0.01$, and $\$ P<0.001$ versus controls; $\# P<0.05$, ${ }^{\# \#} P<0.01$ versus MHC-Pparg mice.

mitate oxidation were not increased in MHC-Pparg mice (Figure $5 \mathrm{~A})$. However, palmitate oxidation rates were increased by $27.3 \%$ and 32.5\% in MHC-Pparg/Ppara-- mice versus controls and MHCPparg hearts, respectively $(P<0.05) . \mathrm{MVO}_{2}$ was reduced by $31 \%$ in $P_{\text {para }}{ }^{-/}$hearts relative to control hearts $(P<0.001$, Figure 5B) and was unchanged in MHC-Pparg hearts. However, PPAR $\alpha$ deficiency increased $\mathrm{MVO}_{2}$ by $18 \%$ in MHC-Pparg hearts compared with controls $(P<0.001)$. Cardiac efficiency, which reflects work performed per unit of oxygen consumed, was reduced in MHC-Pparg hearts because cardiac work was reduced despite "normal" levels of $\mathrm{MVO}_{2}$ (Figure 5C). In MHC-Pparg/Ppara-/ hearts, although cardiac efficiency was similar to that in MHC-Pparg hearts, cardiac power was increased by $49 \%$ relative to MHC-Pparg $(P<0.001$, Figure 5D). Thus, MHC-Pparg/Ppara-/- hearts utilized more oxygen due to greater FAO but had improved function. And, at least under nonischemic conditions, MHC-Pparg/Ppara-/- mice had improved cardiac function associated with greater FAO. This was associated with increased mitochondrial mass as estimated by the ratio of the mitochondrially encoded gene ATPase 6 to $\beta$-actin and mitochondrial transcription factor A (mtTFA) to $18 \mathrm{~s}$ (Figure 5, E and $\mathrm{F}$ ). There was no change in mitochondrial function detected in permeabilized cardiac fibers (Supplemental Figure 4, A-C).

Gene expression in MHC-Pparg/Ppara-/-mice. We determined whether reduction of PPAR $\alpha$ expression would reduce PPAR $\alpha$ target gene expression in the MHC-Pparg mice. We unexpectedly found an increase in PPAR target gene expression in MHC-Pparg/ Ppara-l- mice (Figure 6 and Supplemental Table 1); mRNAs were increased for genes mediating lipid uptake, synthesis, oxidation, lipolysis, and storage, such as fatty acid translocase (Cd36), fatty acid synthase (Fasn), acyl-CoA oxidase ( $A O X)$, adipose TAG lipase
$(A t g l)$, adipose differentiation related protein $(A d r p)$, and diacylglycerol acyltransferase1 (Dgat1). The increase in LCCoA content was associated with increased mRNA expression of long-chain fatty acyl-CoA synthetase (Acsl4), an enzyme expressed in the heart peroxisome (31). However mitochondrial acyl-CoA thioesterase 2 (Acate2), an enzyme that catalyzes the hydrolysis of acyl-CoAs to the FFA and coenzyme A (CoASH), was also increased in MHCPparg/Ppara /- mice (Supplemental Table 1). Expression of PPAR $\gamma$ coactivator $1 \alpha(P g c-1 \alpha)$ and oxidative phosphorylation-related (OXPHOS) genes were unchanged by crossing with $\mathrm{Ppara}^{-/}$mice (Supplemental Table 1). Additionally, PPAR $\alpha$ deficiency dramatically increased cellular retinol-binding protein III (CRBPIII) expression in MHC-Pparg mice (5.91-fold, $P<0.05$ ). In contrast, there was no change in genes regulating glucose metabolism. Thus, the absence of PPAR $\alpha$ appeared to increase the efficacy of the MHCPparg transgene in driving expression of PPAR target genes in the cardiomyocyte. PPAR $\alpha$ deficiency did not alter endogenous PPAR $\delta$ and PPAR $\gamma$ expression (Supplemental Figure 5).

Because loss of PPAR $\alpha$ was, curiously, associated with greater expression of several PPAR $\gamma$ downstream genes, we tested the hypothesis that PPAR $\alpha$ blocked the actions of MHC-Pparg by treating MHC-Pparg mice with the potent PPAR $\alpha$ agonist WY14,643 . Ten days of WY-14,643 treatment decreased plasma TAG without altering FFA or glucose levels (Figure 7A). Heart tissue oil red O staining showed reduced heart neutral lipid in WY-14,643treated MHC-Pparg mice (Figure 7B). Expression of PPAR $\gamma$ downstream targets Cd36, Fasn, and CRBPIII (32) was reduced compared with that in nontreated MHC-Pparg mice. FAO and mitochondrial biogenesis genes, such as $L c a d, U c p 3$, and $P g c-1 \alpha$, were also reduced with WY-14,643 treatment. However, expression of Cpt1, 

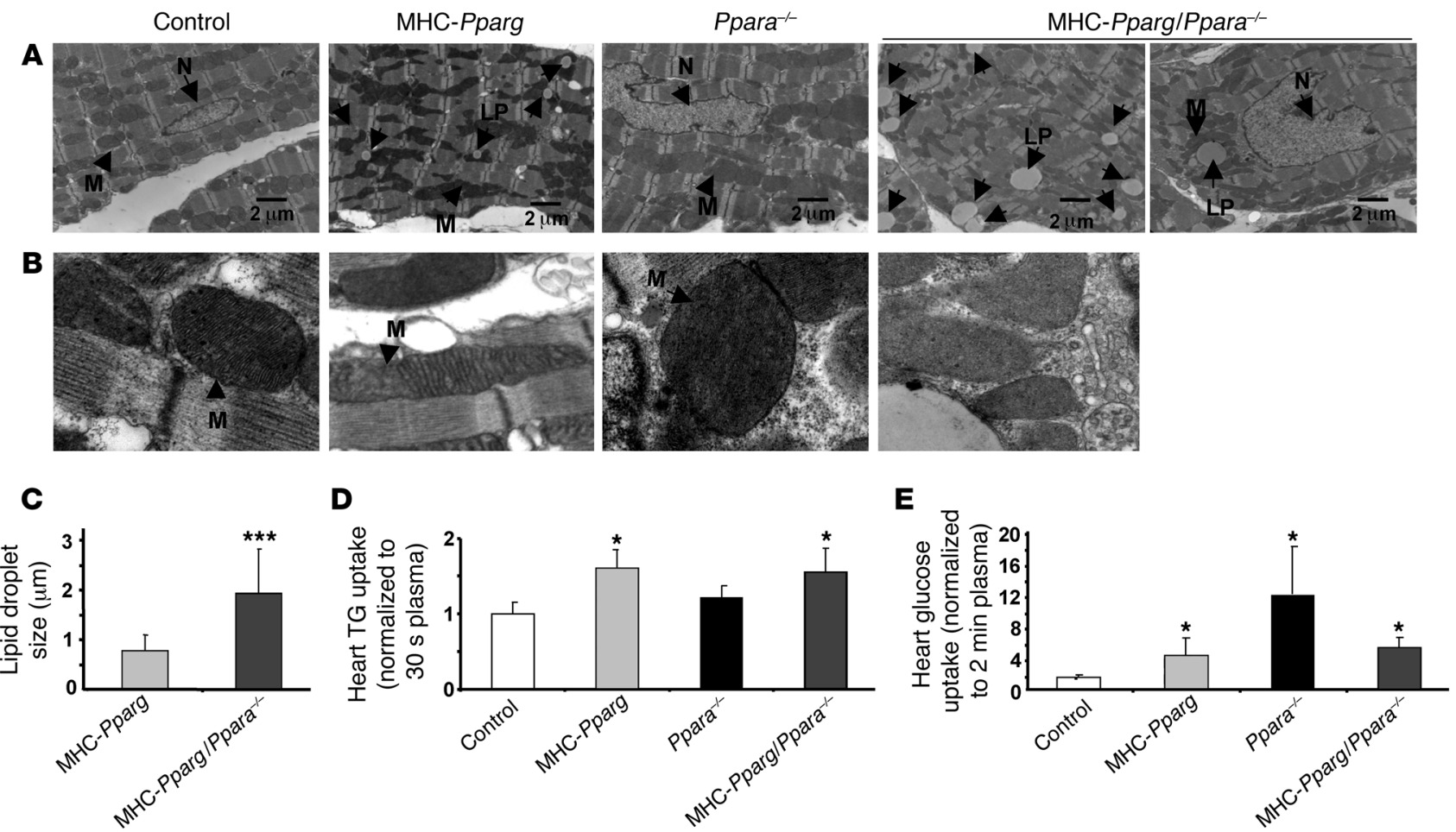

Figure 4

Increase in heart lipid droplet size and TG uptake in MHC-Pparg/Ppara-/- mice. (A) Increase in lipid droplets within the sarcoplasm of cardiomyocytes in both MHC-Pparg and MHC-Pparg/Ppara ${ }^{-/}$mice (original magnification, $\times 5,000$ ). Increase in lipid droplet size (right 2 panels) and larger lipid droplets in MHC-Pparg/Ppara ${ }^{-/}$surrounded by mitochondria (far right). Scale bars: $2 \mu \mathrm{m}$. (B) In MHC-Pparg heart mitochondria, the cristae were disrupted (original magnification, $\times 50,000$ ). (C) The lipid droplet size was determined by randomly counting 50 lipid droplets, and average size is shown. (D) Cardiac TG-VLDL uptake and (E) 2-deoxy-D-[ $\left.{ }^{3} \mathrm{H}\right]$ glucose uptake. LP, lipid droplet; M, mitochondria; N, nucleus. Data are shown as mean \pm SD. ${ }^{*} P<0.05,{ }^{* *} P<0.001$ versus MHC-Pparg mice.

$A O X$, and Dgat1 was not altered by WY-14,643 treatment in MHCPparg mice (Figure 7C). These data suggested that PPAR $\gamma$ activity may be regulated by PPAR $\alpha$.

Evidence that lipid redistribution alters intracellular lipid signaling and reduces apoptosis and ROS. The pattern of intracellular lipid storage relates to the metabolic characteristics of cells; larger droplets have reduced total surface area and are likely to reflect more inert lipid storage (33). We then studied the reasons for the redistribution of cellular lipids. Adrp, perilipin 4, and cell death-inducing DFFA-like effector c (Fsp27) are associated with the surface of intracellular lipid droplets and regulate their formation and mobilization (33-35) and are regulated by PPAR $\gamma$ (36). mRNA expression of $A d r p$, perilipin 4, and Fsp27 was upregulated in MHC-Pparg mice by crossing with $\mathrm{Ppara}^{-/-}$mice (Figure 6, Supplemental Table 1, and Supplemental Figure 6).

Several intracellular lipids are potent signaling molecules and are thought to function as activators of classical PKCs $(37,38)$. To determine whether lipid redistribution led to altered intracellular lipid activation, we measured heart PKC activation. MHC-Pparg increased membrane PKC $\alpha$ and PKCD. This increase was ameliorated in the MHC-Pparg/Ppara-- mice (Figure 8A). Thus, the altered lipid distribution changed lipid activation of this potentially toxic pathway.

Greater FAO is associated with more ROS production (39) and in some situations cellular apoptosis (40). However, TUNEL-positive myocytes were reduced in MHC-Pparg/Ppara-/- compared with MHC-Pparg mice, and expression of the apoptosis-related protein
Bax and p-JNK was decreased in the heart of MHC-Pparg/Ppara-mice (Figure 8, B-D). Heart tissue intracellular $\mathrm{O}_{2}^{-}$levels measured using dihydroethidium and fluorescence staining were increased in MHC-Pparg mice, but this was markedly attenuated in MHCPparg/Ppara ${ }^{--}$mice (Figure 9A). Mitochondrial and ER stress markers prohibitin and protein disulfide isomerase (PDI) $(41,42)$ were also decreased in the MHC-Pparg/Ppara-/- hearts (Figure 9, $\mathrm{B}$ and $\mathrm{C}$ ). This was associated with increased expression of Sod2 but no differences in expression of Gpx1, catalase, Sod1, and Ucp2 between MHC-Pparg and MHC-Pparg/Ppara-- mice. Expression of cardiac hypertrophic markers and apoptosis-related genes, such as natriuretic peptide precursor type B (BNP), serine hydrolase-like (Serh1), DNA damage-inducible transcript 3 (chop), and caspase-6, was significantly decreased in MHC-Pparg/Ppara ${ }^{-/}$mouse hearts (Figure 6B, Figure 9D, and Supplemental Table 1).

\section{Discussion}

Lipotoxicity-induced organ dysfunction is becoming an increasingly common cause of human disease. In the heart, an organ that primarily uses lipids as a source of energy, lipid accumulation assessed as TAG content correlates with reduced function $(4,5,43$, 44). This relationship is found with obesity, metabolic syndrome, and diabetes (6). The pathological pathways linking cellular lipids to cardiac dysfunction are obscure. Our objective was to determine whether excess FAO, one putative cause of lipotoxicity, was corrected in the MHC-Pparg hearts by reduced PPAR $\alpha$ expression and 
A

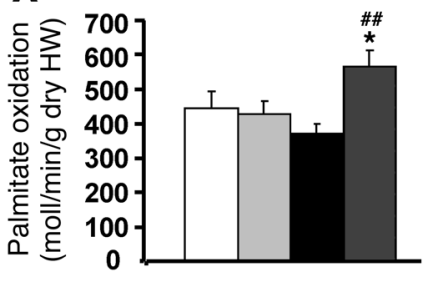

D

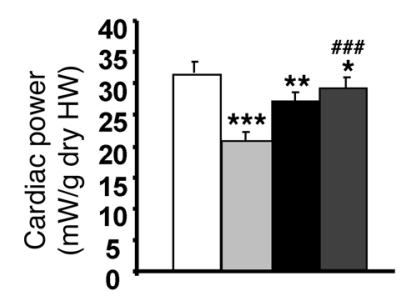

B

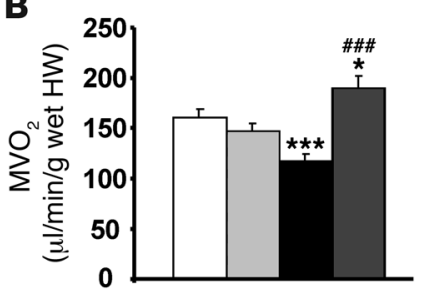

$\mathbf{E}$

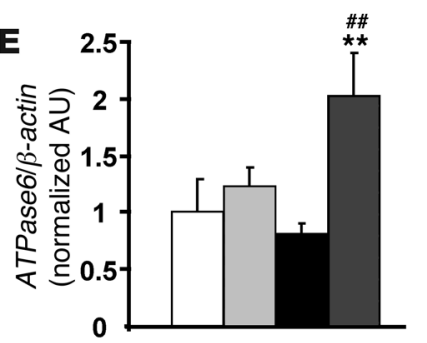

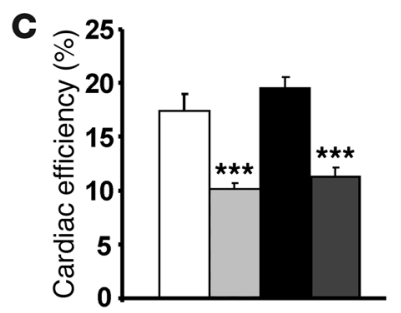

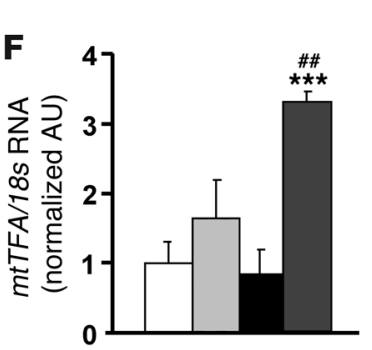

$\square$ Control

$\square$ MHC-Pparg

Ppara ${ }^{-\prime}$

$\square$ MHC-Pparg/Ppara ${ }^{-1}$

\section{Figure 5}

Determination of cardiac lipid oxidation and mitochondria DNA number. (A) Myocardial palmitate oxidation, (B) myocardial oxygen consumption, (C) cardiac efficiency, and (D) cardiac power in isolated working hearts $(n=4-5)$. (E) Heart mitochondrial DNA was quantified by calculating the ratio of mitochondrial gene copy number (ATPase6) to nuclear gene copy number ( $\beta$-actin) $(n=7)$. (F) Heart mtTFA mRNA expression. Data

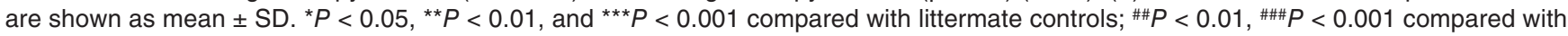
MHC-Pparg mice. HW, heart weight.

whether this would also correct heart dysfunction. By performing a cross of MHC-Pparg onto the Ppara ${ }^{-/}$background, we rescued the heart dysfunction and uncovered a number of fundamental issues related to the causes of heart lipotoxicity. We clearly show that several factors previously implicated as causes of lipotoxicity were not corrected in this model. These include FAO and TAG storage, neither of which were decreased in the MHC-Pparg/Ppara-/- hearts. In addition, there were no major changes in DAG or ceramide levels. Therefore, although loss of PPAR $\alpha$ improved heart function and normalized 5-month survival in the MHC-Pparg mice, it did not do this by the expected mechanisms.

Why did the hearts improve? What was altered was the distribution and size of the lipids: the droplets were significantly larger. Macro- versus microsteatosis has been associated with less dysfunction in the liver (45). Membrane-associated (active) PKC $\alpha$ and PKC $\delta$ were both reduced by the PPAR $\gamma / \mathrm{Ppara}^{-/-} \mathrm{cross}$, an indication that the redistribution of stored lipid was associated with reduced lipid signaling. MHC-Pparg/Ppara/- hearts had reduced levels of acylcarnitines. High cardiac tissue levels of acylcarnitine can disrupt biomembranes through nonspecific detergent actions, induce electrophysiological alterations, and inhibit several critical enzyme systems (46-48). Because LCCoA was increased in the rescued mice, this lipid pattern suggests that the rescued mice more efficiently oxidized acylcarnitines and did not convert unused FAs to this potentially harmful lipid. mRNA levels of Atgl and Dgat1 were increased in the rescued MHC-Pparg/Ppara-/- hearts. In other situations, Dgat1 $(49,50)$ and $\operatorname{Atgl}(51,52)$ expression correlates with increased FAO, and transgenic expression of either of these enzymes relieves lipotoxicity in the heart (53). Thus, we speculate that it is preferable to shunt FAs to the lipid droplet and then allow TAG hydrolysis to oxidative substrates, rather than directly convert internalized FAs into LCCoA and acylcarnitines.

Cardiac muscle is the most energy-requiring tissue in the body and primarily uses FAs and to a great extent lipoprotein-derived
FAs (54). It has been suggested that reduction of FAO might be beneficial in that glucose oxidation is a less oxygen-requiring event. Theoretically, such a switch in substrate use would be especially important in the setting of ischemia and has led to the development of FA inhibitors as a treatment for angina (55). In models of lipotoxicity and hypertrophy, it has also been argued that excess FAO is harmful $(4,56)$. In contrast, high-fat diets are not harmful in rat models of cardiac hypertrophy (57) and in human heart failure (58). Greater FAO in this and one other model (53) of lipotoxicity is clearly not toxic.

Although cardiac toxicity especially in diabetic models is associated with defective mitochondrial function due to mitochondrial uncoupling (59), we found no evidence for changes in mitochondrial function. It should be noted that FFA levels were increased in the PPAR $\alpha$ knockout. While this could have supplied more FA to the hearts, the isolated perfused heart studies and gene expression studies clearly show that MHC-Pparg/Ppara-/- hearts were primed to oxidize more FAO.

Prohibitin is a mitochondrial marker protein localized in the inner membrane of mitochondria. It responds to mitochondrial stress and is induced by metabolic stress caused by an imbalance in the synthesis of mitochondrial- and nuclear-encoded mitochondrial proteins (41). MHC-Pparg heart sections had much more intense staining for prohibitin compared with those from control mice. PPAR $\alpha$ deficiency markedly reduced the staining in MHC-Pparg mice. These data indicate that, in MHC-Pparg/ $\mathrm{Ppara}^{-/}$mice, the improved cardiac function was associated with reduced mitochondrial stress.

MHC-Pparg/Ppara-/- hearts had less ROS, reduced ER stressrelated proteins, and less apoptosis. PDI, a very abundant protein in the ER, is an essential folding catalyst and chaperone of the ER $(60,61)$. There was a large increase in PDI staining in hearts from MHC-Pparg compared with control mice, but this was reduced with PPAR $\alpha$ deficiency. These data suggested that MHC-Pparg 

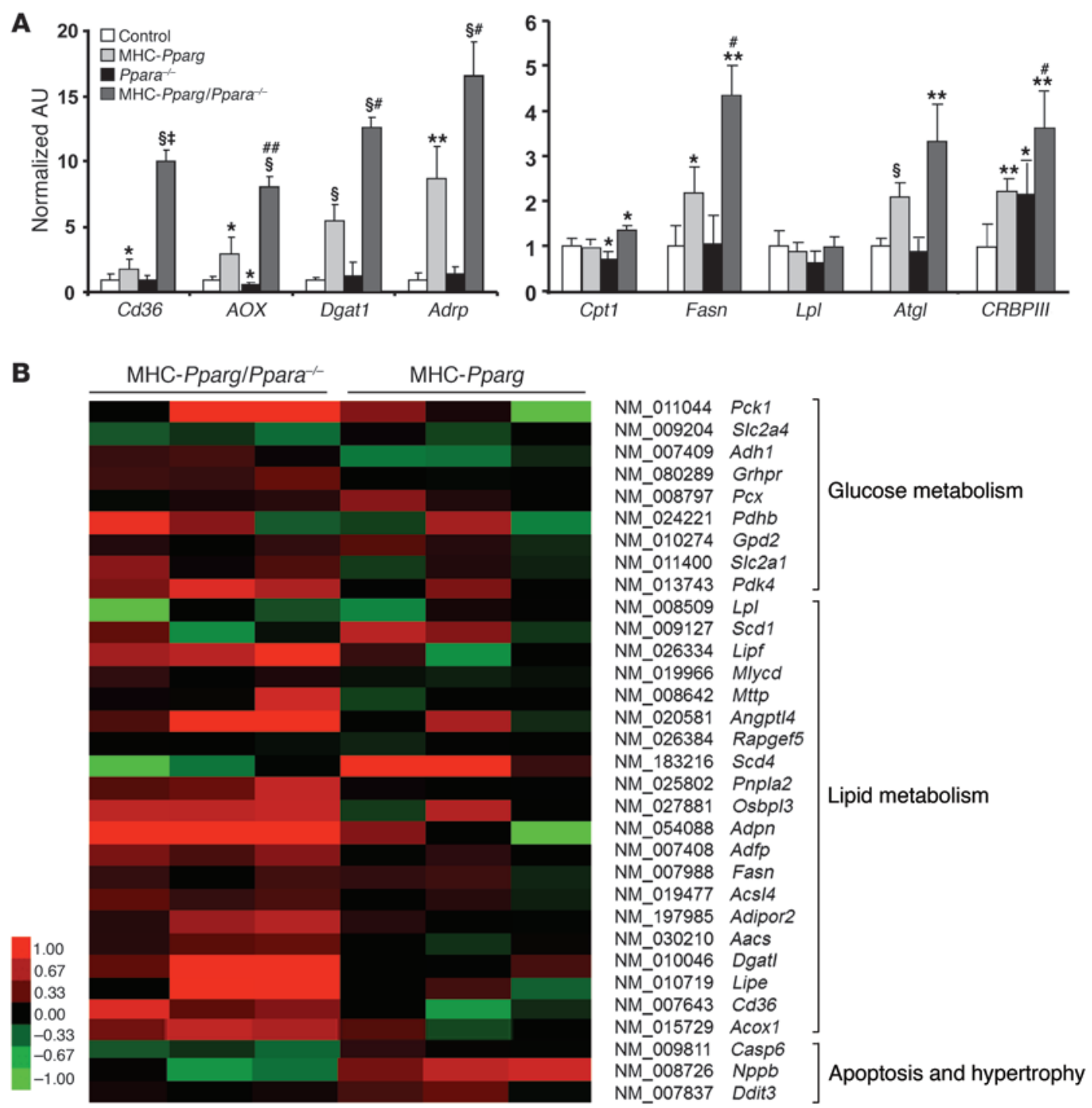

\section{Figure 6}

Heart tissue mRNA expression. (A) qRT-PCR analysis of mRNA expression using gene-specific primers. Data were normalized to $18 \mathrm{~s}$ rRNA. Values represent fold change relative to wild-type controls, which was set as $1(n=5-8)$. Data are shown as mean \pm SD. ${ }^{*} P<0.05$, ${ }^{\star \star} P<0.01$, and $\$ P<0.001$ compared with controls; $\# P<0.05$, \#\# $P<0.01$, and $¥ P<0.001$ compared with MHC-Pparg mice. (B) Clustering of gene expression in $\mathrm{MHC}-\mathrm{Pp}$ arg and $\mathrm{MHC}-\mathrm{Pp}$ parg/Ppara ${ }^{-/-}$mice. Clustering was performed using centered correlation as distance measure and average linkage as method. For the color bar scale, the numeric value is the gene-specific $\log _{10}$ difference in probe intensity from median probe intensity of all 6 samples.

mice had more PDI protein to catalyze disulfide formation, which protects against protein misfolding in the ER.

Although our focus was primarily on cardiac function, we also uncovered evidence that at least in the heart, PPAR $\gamma$ and PPAR $\alpha$ are not equivalent and their activation is not always synchronous. Unexpectedly, MHC-Pparg/Ppara-/- hearts had greater expression of several genes regulating FA metabolism. This was surprising, and we then questioned whether in the heart PPAR $\gamma$ was a more potent activator of these genes and whether its actions in the MHC-Pparg mice were actually blocked by the presence of PPAR $\alpha$. We tested this by treating MHC-Pparg mice with the potent agonist WY-14,643. Expression of two PPAR $\gamma$ downstream genes, Cd36 and CRBPIII, was reduced by this agonist; a cardiac PPAR $\alpha$ target, Cpt1, was induced. Therefore, it appears that not all PPAR activa- tion is of similar potency and PPAR $\gamma$ actions were diminished by the actions of PPAR $\alpha$. The molecular events for this phenomenon - competition for promoter binding sites, coactivators or repressors, or lipid agonists - are under study.

Because all three members of the PPAR gene family have been overexpressed in the heart, their functional and genetic effects can be compared. PPAR $\alpha$, which increases FAO, leads to greater lipid uptake in the heart, and this lipid uptake is out of proportion to the induction of oxidation (4). PPAR $\alpha$ is most highly expressed in the liver, a tissue in which lipid uptake that exceeds oxidation can be compensated by TAG secretion in lipoproteins. In contrast, hearts from MHC-Ppard mice have increased FAO but no cardiac dysfunction or accumulation of lipid; oxidation and lipid uptake are balanced (62). Thus, this gene expression program is 


\section{A}

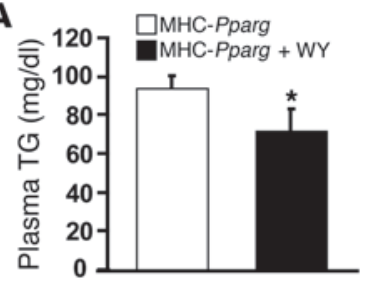

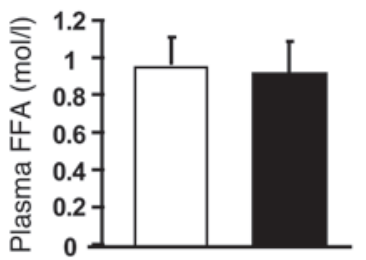

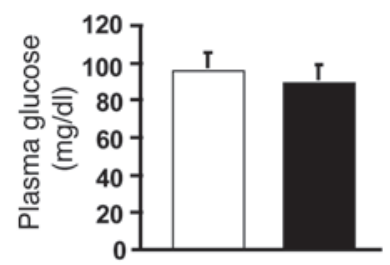

\section{Figure 7}

MHC-Pparg mice with or without WY14,643 treatment. (A) Plasma TG, FFA, and glucose concentrations in the mice. (B) Oil red $\mathrm{O}$ staining of hearts from 3-month-old MHC-Pparg mice with or without WY-14,643 treatment (original magnification, $\times 200$ ). (C) qRT-PCR analysis of cardiac mRNA expression in MHC-Pparg mice with or without WY14,643 treatment ( $n=6-7$ per group). The results were repeated in 2 independent experiments. Data are shown as mean \pm SD. ${ }^{*} P<0.05,{ }^{* *} P<0.01$, $\S P<0.001$ compared with nontreated MHC-Pparg mice.

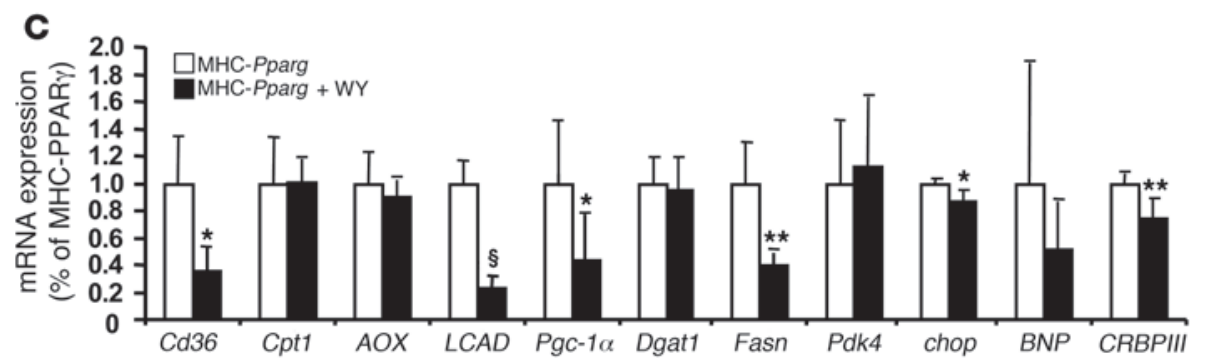

most suitable for muscles in which greater oxidation allows for more energy production but without leading to toxicity, e.g., with exercise (63). Like PPAR $\alpha$, PPAR $\gamma$ increases lipid uptake in excess of that needed for oxidation. However, the MHC-Pparg transgene does not increase Pdk4 and is not associated with reduced glucose uptake (15). These changes in gene expression are appropriate to increase lipid stores in adipose but can lead to toxicity in muscles. The overlapping and competing actions of PPAR transcription factors may be central to metabolic regulation.

Although PPAR $\gamma$ is expressed in the heart at much lower levels than in adipose $(64,65)$, use of agents that lead to marked induction of this transcription factor are associated with cardiac toxicity (66). In humans, several PPAR $\gamma$ agonists increase the incidence of clinical heart failure $(67,68)$. PPAR $\gamma$ agonists have a number of actions, including salt and water retention and vasodilatation (69), and these actions or direct cardiac toxicity might be responsible for the adverse effects in humans. Hearts of patients with metabolic syndrome have greater lipid accumulation; these hearts also have increased expression of PPAR $\gamma$ (70). Thus, cardiac PPAR $\gamma$ expression is either responsible for or coincidental with cardiac lipid content in humans.

Reduced cardiac function with increased lipid is found in patients with obesity (71) and diabetes (72). Aside from lipid, heart dysfunction with diabetes is postulated to involve microvascular disease, glycosylation of intracellular proteins, and greater FAO (73). Models in which lipid accumulation is increased solely in the heart are likely to be more flagrant representations of human cardiac lipotoxicity and provide the opportunity to dissect the roles of lipids and FAO in cardiac dysfunction exclusive of generalized metabolic derangements. Our data show that processes thought to cause lipotoxicity can be illustrated and eliminated by genetic methods. Excess FAO was not corrected in the MHC-Pparg/Ppara-/mice. Thus, this is not a likely cause of toxicity. Rather, further induction of FAO appears to be protective. Stores of TAG, although a marker of the disease, are also not a cause of toxicity. Improved cardiac function correlated with a redistribution of intracellular lipids and reduced activation of PKCs, despite unchanged levels of total DAG and ceramide. Because this cross-corrected many processes associated with lipotoxicity - ROS, apoptosis, and PKC signaling were reduced - a single specific cause and toxic pathway cannot be discerned. Reduced lipid uptake into the heart corrects cardiac toxicity in MHC-Ppara mice $(74,75)$ presumably by reducing accumulated lipid. Our study underscores another option for treatment of lipotoxic cardiomyopathy and perhaps other lipotoxic diseases, namely increased cellular lipid oxidation and/or greater storage of relatively "nontoxic" lipid moieties.

\section{Methods}

Animal studies and generation of MHC-Pparg/Ppara-/- mice. The high-expressing MHC-Pparg mouse line (15) in a mixed C57BL $/ 6 \times \mathrm{CBA} / \mathrm{J}$ background was backcrossed with C57BL/ 6 wild-type mice (The Jackson Laboratory) for 10 generations, resulting in greater than $99 \%$ C57BL/6 purity. MHCPparg mice were then crossed twice into the $P$ para ${ }^{-/}$(The Jackson Laboratory) background, resulting in MHC-Pparg/Ppara-/ offspring. Studies of gene expression, histology, lipid analysis, and cardiac function by echocardiography were performed using mice fed chow diets with overnight fasting. All animal experiments were approved by the Institutional Animal Care and Use Committee of the Columbia University Medical Center.

Plasma and heart lipids. Blood from fasted (16 hours) mice was collected from the retro-orbital plexus for the measurement of plasma total cholesterol (TC), TAG, and FFAs. For measurement of tissue lipids, hearts were perfused with $\mathrm{PBS}$ and homogenized at $4^{\circ} \mathrm{C}$ in $1 \mathrm{M} \mathrm{NaCl}$ buffer containing 
A

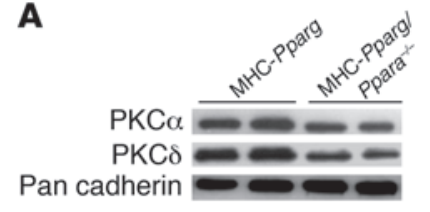

B

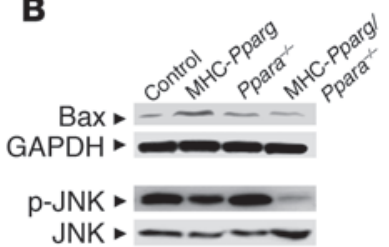

C
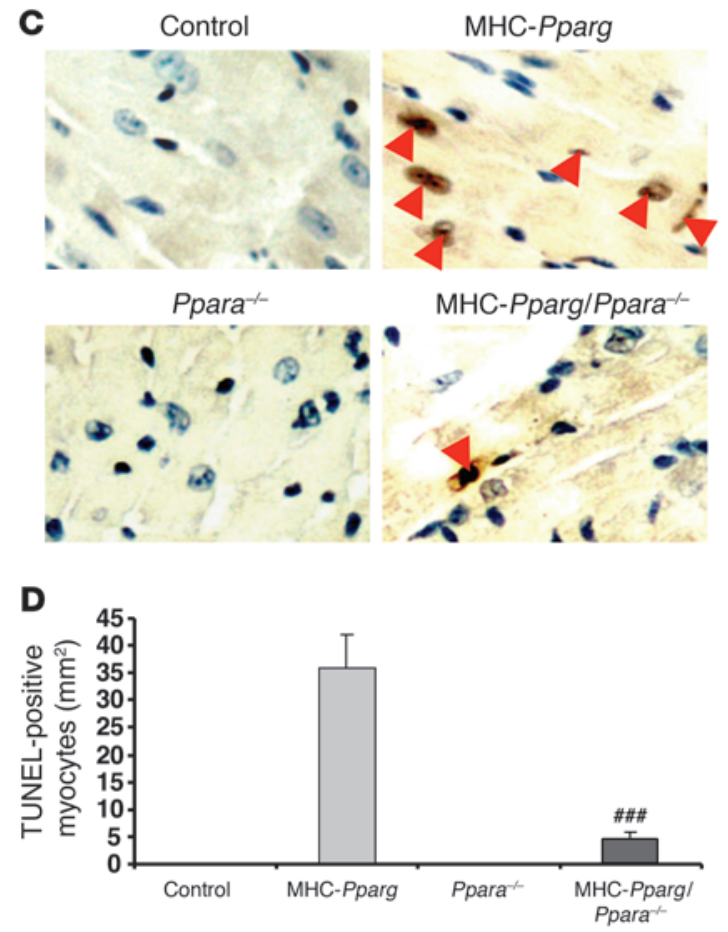

lipase inhibitors to prevent TAG hydrolysis. Lipids were extracted from heart tissues $(50 \mathrm{mg})$ according to methods modified from that of Folch et al. (76). The dried lipids were solubilized in PBS containing $2 \%$ Triton X-100. Heart and plasma TC, TAG, and FFAs were measured enzymatically using an Infinity kit (Thermo Electron Corp.) and a NEFA C kit (Wako). Heart tissue FA analysis was performed using gas chromatographic methods as described previously (77).

Western blot analysis. Heart tissues from 3-month-old mice were prepared, and whole-cell proteins were isolated using RIPA lysis buffer (sc-24948; Santa Cruz Biotechnology Inc.). Thirty micrograms of nuclear proteins were subjected to Western blot analysis with the following antibodies: PPAR $\gamma$ (sc-7196; 1:100 dilution) and PPAR $\alpha$ (sc-9000; 1:200 dilution) from Santa Cruz Biotechnology Inc.; Bax (ab54481; 1:500 dilution), PKC $\alpha$ (ab4142; 1:1,000 dilution), PKC (ab4143; 1:1,000 dilution), and Gapdh (ab9485; 1:5,000 dilution) from Abcam Inc. Immunoreactive bands were visualized using SuperSignal West Pico Chemiluminescent Substrate (Pierce). The bands were quantified by densitometry using Molecular Analysis Software (Bio-Rad).

Quantitative real-time RT-PCR analysis. Total RNA was prepared using a Pure Link Micro-to-Midi Total Purification System kit (Invitrogen). One microgram of RNA was initially treated with DNase I (Invitrogen) for 15 minutes. The RNA samples were then reverse transcribed using the ThermoScript RT-PCR Kit (Invitrogen). Quantitative real-time RT-PCR (qRT-PCR) was performed using an ABI 7700 (Applied Biosystems). Amplification was performed using SYBR Green PCR Master Mix (Applied Biosystems). Primers used for PCR amplification are listed in Supplemental Table 2. Analysis

\section{Figure 8}

Cardiac PKC content and apoptosis-related proteins. (A) Representative Western blot image of membrane PKC $\alpha$ and PKC $\delta$ content. (B) BAX and p-JNK proteins in the heart. Pan-cadherin, Gapdh, and total JNK are shown as controls. (C) Cardiac ventricular tissues were stained for DNA fragmentation by TUNEL protocol (original magnification, $\times 200$ ). Apoptotic cardiomyocytes containing fragmented nuclear chromatin exhibited dark brown nuclear staining (arrows). (D) The TUNEL-positive myocytes were counted and expressed as the number of TUNEL-positive myocytes per millimeter squared tissue area. Data are shown as mean \pm SD. $\# \# P<0.0001$ compared with MHC-Pparg mice.

was performed using Sequence Detection Software (Applied Biosciences). Standard curves were generated using undiluted and diluted (1:10, 1:100, and 1:1,000) cDNA samples from heart tissue. Correlation coefficients were 0.98 or greater. Data were normalized with 18 s rRNA.

Analysis of RNA microarray data. Mouse heart tissue samples in RNA later were sent to Ocean Ridge Biosciences (ORB) for analysis using mouse exonic evidence-based oligonucleotide (MEEBO) microarrays (lot 20788). MEEBO microarrays were printed by Microarrays Inc. and contained 38,083 70-mer oligonucleotides probes complementary to constitutive exons of most mouse genes, as well as alternatively spliced exons, and control sequences. For statistical analysis, samples were binned in two treatment groups (MHC-Pparg and MHC-Pparg/Ppara-/-). The $\log _{2}-$ transformed and normalized spot intensities for the 19,420 detectable probes were examined for differences between the treatment groups by 1-way ANOVA using National Institute on Aging (NIA) Array Analysis software. This ANOVA was conducted using the Bayesian error model and 10 degrees of freedom. Statistical significance was determined using the false discovery rate (FDR) method. It is the proportion of false positives among all probes with $P$ values lower or equal to the $P$ value of the probes that we considered significant. FDR is an intermediate method between the $P$ value and Bonferroni correction (multiplying $P$ value by the total number of probes). The equation is:

$$
F D R_{r}=\min _{i \geq r}\left[\frac{p_{i} N}{i}\right]
$$

(Equation 1)

where $r$ is the rank of a probe ordered by increasing $P$ values, $p_{i}$ is the $P$ value for probe with rank $i$, and $N$ is the total number of probes tested. FDR value increases monotonously with increasing $P$ value.

Measurement of mitochondrial DNA. Mitochondrial DNA was quantified by calculating the ratio of mitochondrial gene copy number (ATPase6) to nuclear gene copy number $(\beta$-actin) $(78,79)$. Heart DNA was extracted from frozen tissue with a DNeasy tissue kit (QIAGEN).

Histological analysis. Neutral lipids were assessed in hearts taken from 16-hour-fasted mice perfused with PBS. The hearts were embedded in Tissue-Tek Optimal Cutting Temperature compound (Sakura). Midventricular sections of myocardium ( $6 \mu \mathrm{M}$ in thickness) were stained with oil red $\mathrm{O}$ and $\mathrm{H} \& \mathrm{E}$ and counterstained with hematoxylin. We used dihydroethidium (D23107; Invitrogen) to examine the superoxide in the frozen heart tissue sections. Immunohistochemical staining techniques were used on frozen sections to examine the mitochondria-associated protein prohibitin (ab1836; Abcam). An Endoplasmic Reticulum Labeling Kit was used for detecting ER-associated PDI with a primary antibody directed against PDI antibody and an Alexa Fluor 488 dye-labeled secondary antibody (S34252; Invitrogen). Apoptotic cardiomyocytes were stained by a TUNEL protocol according to the manufacturer's specifications (R\&D Systems). The data were quantified by counting TUNEL-positive myocytes per square millimeter cellular area. 

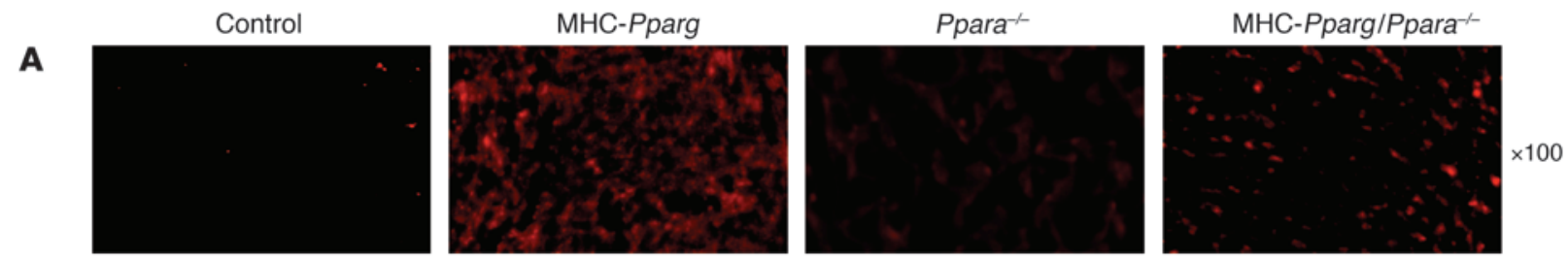

B
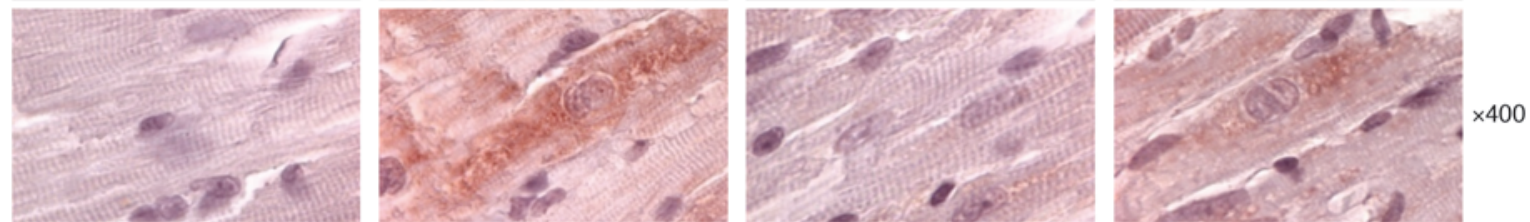

C
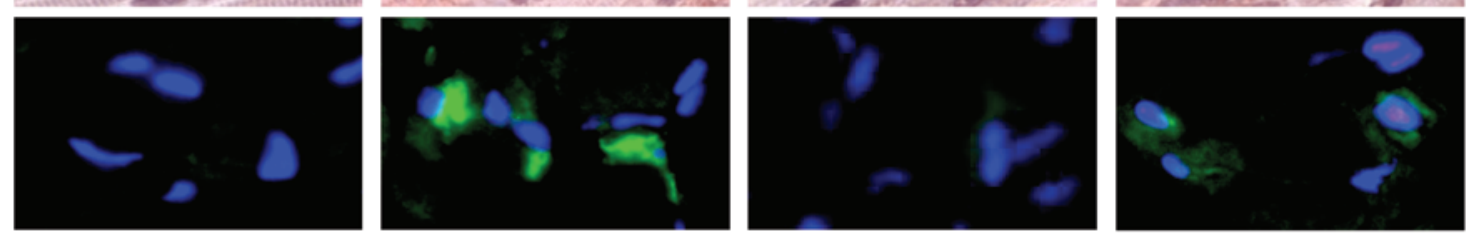

$\times 400$
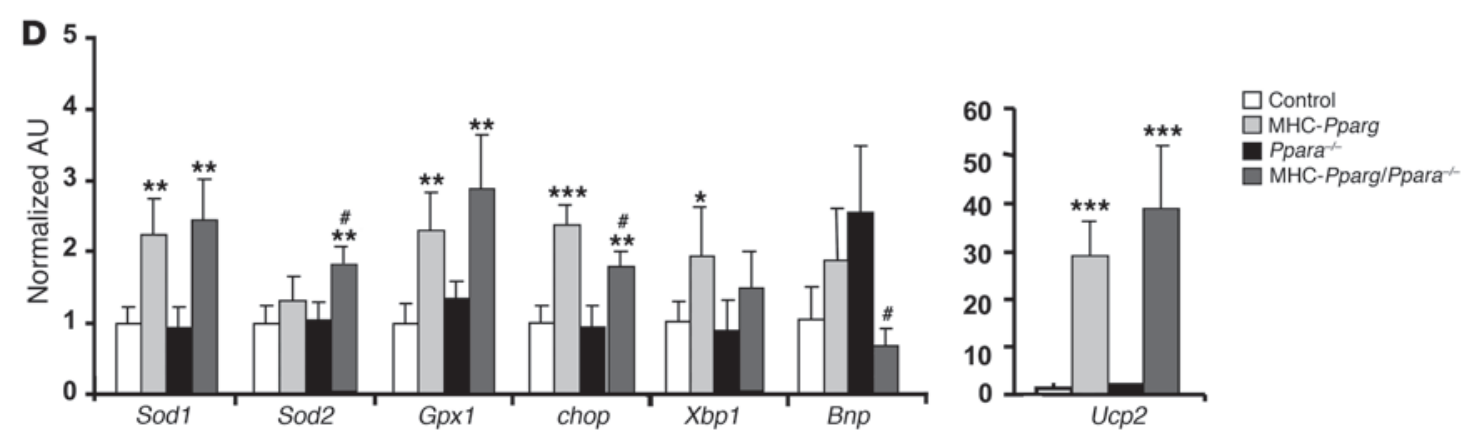

Figure 9

Detection of ROS production and of mitochondrial and ER stress in heart tissues. (A) Histological analysis of heart tissues using dihydroethidium staining to detect ROS (original magnification, $\times 100$ ). (B) Mitochondrial stress was detected by immunohistochemical staining of heart tissue sections with antibodies against prohibitin protein (original magnification, $\times 400$ ). (C) Immunofluorescence analysis of the heart tissues using disulfide isomerase antibody (original magnification, $\times 400$ ). (D) qRT-PCR analysis of heart gene expression. Data were normalized to 18s RNA. Values represent fold change relative to wild-type controls, which was set as $1(n=5-8)$. Data are shown as mean $\pm \mathrm{SD}$. ${ }^{*} P<0.05$, ${ }^{* *} P<0.01$, and ${ }^{* * *} P<0.01$ compared with littermate controls; $\# P<0.05$ compared with MHC-Pparg mice.

Echocardiographic analysis. Transthoracic echocardiography was performed in conscious mice using a high-resolution imaging system with a 30-MHz imaging transducer (Vevo 770; VisualSonics). Each parameter was measured using M-mode view. Percent fractional shortening (\%FS) was calculated as follows: $\% \mathrm{FS}=(\mathrm{LVDd}-\mathrm{LVDs}) / \mathrm{LVDd} \times 100$, where LVDd is left ventricular diastolic dimension and LVDs is left ventricular systolic dimension (80).

Electron microscopy. Left ventricles from 3-month-old mice were fixed with $2.5 \%$ glutaraldehyde in $0.1 \mathrm{M}$ Sorensen's buffer $(0.2 \mathrm{M}$ monobasic phosphate/0.2 M dibasic phosphate, $1: 4 \mathrm{vol} / \mathrm{vol}$; $\mathrm{pH} 7.2$ ), postfixed in osmium tetroxide, and embedded in EPON 812 (Electron Microscopy Sciences). Ultrathin sections were stained with uranyl acetate and lead citrate and examined under a JEM-1200ExII electron microscope (JEOL).

Uptake of VLDL and glucose. Human VLDL was isolated from normal subjects by sequential ultracentrifugation and was labeled with [carboxyl$\left.{ }^{14} \mathrm{C}\right]$ triolein (PerkinElmer) via cholesterol ester transfer protein as previously described (81). Sixteen-hour-fasted mice were first injected intravenously with $1 \times 10^{6}$ decays per minute (DPM) of 2-deoxy-D- $\left[{ }^{3} \mathrm{H}\right]$ glucose (PerkinElmer). Fifty-five minutes after 2-deoxy-D- $\left[{ }^{3} \mathrm{H}\right]$ glucose injection, $1 \times 10^{6}$ DPM of $\left[{ }^{14} \mathrm{C}-\mathrm{TAG}\right] \mathrm{VLDL}$ was injected. Five minutes after VLDL injection, blood was collected, and the vasculature was thoroughly perfused with
$10 \mathrm{ml}$ PBS via cardiac puncture. Tissues were then excised, and accumulated radioactivity for $\left[{ }^{3} \mathrm{H}\right]$ glucose and $\left[{ }^{14} \mathrm{C}-\mathrm{TAG}\right] \mathrm{VLDL}$ was measured. Amounts of glucose and VLDL injected were adjusted by plasma radioactivity counts at 30 seconds after each injection and were compared with plasma counts at the end of the experiments.

Measurement of palmitate oxidation and $\mathrm{MVO}_{2}$. Cardiac metabolism was measured in isolated working mouse hearts as described previously (30).

Mitochondrial function. Mitochondrial oxygen consumption and ATP production were measured in permeabilized cardiac fibers isolated from 12-week-old wild-type, MHC-Pparg, Ppara-/, and MHC-Pparg/Ppara-/ mice using techniques described previously $(59,82)$.

Ceramide and DAG. Cardiac ceramide and diacylglycerol (DAG) levels were determined using the DAG kinase method as described previously (83). Individual species of DAG and ceramides were measured by liquid chromatography-mass spectrometry as described previously (84).

Immunoblot analysis of PKC contents. Heart tissues $(100 \mathrm{mg})$ from 3-monthold mice were homogenized and extracted in cold $20 \mathrm{mM}$ Tris- $\mathrm{HCl}(\mathrm{pH}$ 8.0), 2 mM EDTA, 2 mM EGTA, 6 mM 2-mercaptoethanol, $0.1 \mathrm{mM}$ sodium vanadate, $50 \mathrm{mM} \mathrm{NaF}$, and complete inhibitor cocktail (Roche). The homogenate was solubilized and centrifuged at $4^{\circ} \mathrm{C}$ for 1 hour at $100,000 \mathrm{~g}$. Separation of cytosol and membrane fraction was done as described pre- 
viously (85). Equivalent amounts of the two fractions were subjected to SDS-PAGE. Proteins were electroblotted onto nitrocellulose membranes, which were probed with rabbit anti-peptide antibodies specific for PKC $\alpha$ and PKC $\delta$ isozymes (Santa Cruz Biotechnology Inc.). PKC isoenzymes were then visualized by incubation of the membrane with enhanced chemiluminescence reagents and exposure to X-ray film. Densitometry of PKC bands was carried out using a Medical Dynamics Personal Densitometer and analyzed using QuantityOne software (Bio-Rad).

PPAR $\alpha$ agonist treatment. PPAR $\alpha$ agonist (WY-14,643) was purchased from Sigma-Aldrich and given as $50 \mathrm{mg} / \mathrm{kg} / \mathrm{d}$ in aqueous hydroxypropyl methylcellulose once daily for 10 days by oral gavage (86).

Statistics. We analyzed data using the Prism software package (GraphPad Software). Comparisons between two groups were performed using unpaired 2 -tailed Student's $t$ tests. All values are presented as mean $\pm \mathrm{SD}$. Differences between groups were considered statistically significant at $P<0.05$.

\section{Acknowledgments}

We thank K.G. Bharadwaj, H. Jiang, and L. Liu for help with the kinetic studies, PCR, and measurement of ceramide and DAG; and Jamie Soto for help with mitochondrial analyses. These studies were supported by grants HL45095, HL73029, P50 HL077113, and U01HL087947 from the National Heart, Lung, and Blood Institute.

Received for publication June 10, 2010, and accepted in revised form July 21, 2010.

Address correspondence to: Ira J. Goldberg, Department of Medicine, Columbia University, 630 West 168th Street, PH10-305, New York, New York 10032, USA. Phone: 212.305.5961; Fax: 212.305.3213; E-mail:ijg3@columbia.edu.
1. Unger RH. Lipotoxic diseases. Annu Rev Med. 2002;53:319-336.

2. Carvajal K, Moreno-Sanchez R. Heart metabolic disturbances in cardiovascular diseases. Arch Med Res. 2003;34(2):89-99.

3. Finck BN, et al. A critical role for PPARalpha-mediated lipotoxicity in the pathogenesis of diabetic cardiomyopathy: modulation by dietary fat content. Proc Natl Acad Sci U S A. 2003;100(3):1226-1231.

4. Finck BN, et al. The cardiac phenotype induced by PPARalpha overexpression mimics that caused by diabetes mellitus. JClin Invest. 2002;109(1):121-130.

5. Schaffer JE. Lipotoxicity: when tissues overeat. Curr Opin Lipidol. 2003;14(3):281-287.

6. Zhou YT, et al. Lipotoxic heart disease in obese rats: implications for human obesity. Proc Natl Acad Sci US A. 2000;97(4):1784-1789.

7. Torffvit O, Agardh C. The prognosis for type 2 diabetic patients with heart disease. A 10-year observation study of 385 patients. J Diabetes Complications. 2000;14(6):301-306.

8. Baidyaroy D, et al. A gene related to yeast HOS2 histone deacetylase affects extracellular depolymerase expression and virulence in a plant pathogenic fungus. Plant Cell. 2001;13(7):1609-1624.

9. Yagyu $H$, et al. Lipoprotein lipase (LpL) on the surface of cardiomyocytes increases lipid uptake and produces a cardiomyopathy. J Clin Invest. 2003;111(3):419-426.

10. Chiu HC, et al. Transgenic expression of fatty acid transport protein 1 in the heart causes lipotoxic cardiomyopathy. Circ Res. 2005;96(2):225-233.

11. Luo J, et al. A protocol for rapid generation of recombinant adenoviruses using the AdEasy system. Nat Protoc. 2007;2(5):1236-1247.

12. van Herpen NA, Schrauwen-Hinderling VB. Lipid accumulation in non-adipose tissue and lipotoxicity. Physiol Behav. 2008;94(2):231-241.

13. Zhang H, Zhang A, Kohan DE, Nelson RD, Gonzalez FJ, Yang T. Collecting duct-specific deletion of peroxisome proliferator-activated receptor gamma blocks thiazolidinedione-induced fluid retention. Proc Natl Acad Sci U S A. 2005;102(26):9406-9411.

14. Vikramadithyan RK, et al. Euglycemic and hypolipidemic activity of PAT5A: a unique thiazolidinedione with weak peroxisome proliferator activated receptor gamma activity. Metabolism. 2000;49(11):1417-1423.

15. Son $\mathrm{NH}$, et al. Cardiomyocyte expression of PPARgamma leads to cardiac dysfunction in mice. J Clin Invest. 2007;117(10):2791-2801.

16. Marfella R, et al. Thiazolidinediones may contribute to the intramyocardial lipid accumulation in diabetic myocardium: effects on cardiac function. Heart. 2009;95(12):1020-1022.

17. Park TS, et al. Ceramide is a cardiotoxin in lipotoxic cardiomyopathy. J Lipid Res. 2008;49(10):2101-2112.

18. Listenberger LL, Ory DS, Schaffer JE. Palmitate-induced apoptosis can occur through a ceramide-independent pathway. J Biol Chem. 2001; 276(18):14890-14895.

19. Nojiri H, et al. Oxidative stress causes heart failure with impaired mitochondrial respiration. J Biol Chem. 2006;281(44):33789-33801.

20. Borradaile NM, Han X, Harp JD, Gale SE, Ory DS, Schaffer JE. Disruption of endoplasmic reticulum structure and integrity in lipotoxic cell death. J Lipid Res. 2006;47(12):2726-2737.

21. Leone TC, Weinheimer CJ, Kelly DP. A critical role for the peroxisome proliferator-activated receptor alpha (PPARalpha) in the cellular fasting response: the PPARalpha-null mouse as a model of fatty acid oxidation disorders. Proc Natl Acad Sci U S A. 1999; 96(13):7473-7478.

22. Peters JM, et al. Alterations in lipoprotein metabolism in peroxisome proliferator-activated receptor alpha-deficient mice. J Biol Chem. 1997; 272(43):27307-27312.

23. Crowder CM. Cell biology. Ceramides - friend or foe in hypoxia? Science. 2009;324(5925):343-344.

24. Menuz V, et al. Protection of C. elegans from anoxia by HYL-2 ceramide synthase. Science. 2009; 324(5925):381-384.

25. Markwell MA, McGroarty EJ, Bieber LL, Tolbert NE. The subcellular distribution of carnitine acyltransferases in mammalian liver and kidney. A new peroxisomal enzyme. J Biol Chem. 1973;248(10):3426-3432.

26. Cases S, et al. Identification of a gene encoding an acyl CoA:diacylglycerol acyltransferase, a key enzyme in triacylglycerol synthesis. Proc Natl Acad Sci U S A. 1998;95(22):13018-13023.

27. Corr PB, Creer MH, Yamada KA, Saffitz JE, Sobel BE. Prophylaxis of early ventricular fibrillation by inhibition of acylcarnitine accumulation. J Clin Invest. 1989;83(3):927-936.

28. Yamada KA, Kanter EM, Newatia A. Long-chain acylcarnitine induces $\mathrm{Ca} 2+$ efflux from the sarcoplasmic reticulum. J Cardiovasc Pharmacol. 2000;36(1):14-21.

29. Varela A, Dalamon V, Testoni G, Carregal M, Cerruti S, Savino EA. Effects of pentanoate, 4-pentenoate, 3-hydroxybutyrate and insulin on the tissuelevels of long-chain acyl CoA and acylcarnitine in the oxygenated and hypoxic rat atria. Arch Physiol Biochem. 1999;107(1):22-26.

30. Mazumder PK, et al. Impaired cardiac efficiency and increased fatty acid oxidation in insulin-resistant ob/ ob mouse hearts. Diabetes. 2004;53(9):2366-2374.

31. Coleman RA, Lewin TM, Van Horn CG, Gonzalez-Baro MR. Do long-chain acyl-CoA synthetases regulate fatty acid entry into synthetic versus degradative pathways? J Nutr. 2002;132(8):2123-2126.

32. Zizola CF, Schwartz GJ, Vogel S. Cellular retinolbinding protein type III is a PPARgamma target gene and plays a role in lipid metabolism. Am J Physiol Endocrinol Metab. 2008;295(6):E1358-1368.

33. Nishino N, et al. FSP27 contributes to efficient energy storage in murine white adipocytes by pro- moting the formation of unilocular lipid droplets. J Clin Invest. 2008;118(8):2808-2821.

34. Miura S, et al. Functional conservation for lipid storage droplet association among Perilipin, ADRP, and TIP47 (PAT)-related proteins in mammals, Drosophila, and Dictyostelium. J Biol Chem. 2002;277(35):32253-32257.

35. Puri V, et al. Cidea is associated with lipid droplets and insulin sensitivity in humans. Proc Natl Acad Sci US A. 2008;105(22):7833-7838.

36. Wolins NE, et al. OXPAT/PAT-1 is a PPAR-induced lipid droplet protein that promotes fatty acid utilization. Diabetes. 2006;55(12):3418-3428.

37. Way KJ, Chou E, King GL. Identification of PKCisoform-specific biological actions using pharmacological approaches. Trends Pharmacol Sci. 2000;21(5):181-187.

38. Way KJ, Katai N, King GL. Protein kinase C and the development of diabetic vascular complications. Diabet Med. 2001;18(12):945-959.

39. Bhaumik P, Koski MK, Glumoff T, Hiltunen JK, Wierenga RK. Structural biology of the thioester-dependent degradation and synthesis of fatty acids. Current Opinion in Structural Biology. 2005;15(6):621-628.

40. Barzilai A, Yamamoto KI. DNA damage responses to oxidative stress. DNA Repair. 2004;3(8-9):1109-1115.

41. Coates PJ, et al. Mammalian prohibitin proteins respond to mitochondrial stress and decrease during cellular senescence. Exp Cell Res. 2001; 265(2):262-273.

42. Azfer A, Niu J, Rogers LM, Adamski FM, Kolattukudy PE. Activation of endoplasmic reticulum stress response during the development of ischemic heart disease. Am J Physiol Heart Circ Physiol. 2006; 291(3):H1411-H1420.

43. Christoffersen $\mathrm{C}$, et al. Cardiac lipid accumulation associated with diastolic dysfunction in obese mice. Endocrinology. 2003;144(8):3483-3490.

44. Chiu HC, et al. A novel mouse model of lipotoxic cardiomyopathy. J Clin Invest. 2001;107(7):813-822.

45. Burt AD, Mutton A, Day CP. Diagnosis and interpretation of steatosis and steatohepatitis. Semin Diagn Pathol. 1998;15(4):246-258.

46. Wu J, Corr PB. Palmitoyl carnitine modifies sodium currents and induces transient inward current in ventricular myocytes. Am J Physiol. 1994; 266(3 pt 2):H1034-H1046.

47. Yamada KA, et al. Cellular uncoupling induced by accumulation of long-chain acylcarnitine during ischemia. Circ Res. 1994;74(1):83-95.

48. Shug AL, Shrago E, Bittar N, Folts JD, Koke JR. Acyl-CoA inhibition of adenine nucleotide translocation in ischemic myocardium. Am J Physiol. 1975;228(3):689-692.

49. Monetti M, et al. Dissociation of hepatic steatosis and insulin resistance in mice overexpressing DGAT in the liver. Cell Metab. 2007;6(1):69-78.

50. Liu L, Zhang Y, Chen N, Shi X, Tsang B, Yu YH. 
Upregulation of myocellular DGAT1 augments triglyceride synthesis in skeletal muscle and protects against fat-induced insulin resistance. J Clin Invest. 2007;117(6):1679-1689.

51. Reid BN, et al. Hepatic overexpression of hormonesensitive lipase and adipose triglyceride lipase promotes fatty acid oxidation, stimulates direct release of free fatty acids, and ameliorates steatosis. J Biol Chem. 2008;283(19):13087-13099.

52. Zimmermann R, et al. Fat mobilization in adipose tissue is promoted by adipose triglyceride lipase. Science. 2004;306(5700):1383-1386.

53. Liu L, et al. DGAT1 expression increases heart triglyceride content but ameliorates lipotoxicity. J Biol Chem. 2009;284(52):36312-36323.

54. Ballard FB, Danforth WH, Naegle S, Bing RJ. Myocardial metabolism of fatty acids. J Clin Invest. 1960;39:717-723.

55. Lopaschuk GD. Treating ischemic heart disease by pharmacologically improving cardiac energy metabolism. Am J Cardiol. 1998;82(5A):14K-17K.

56. Sambandam N, Lopaschuk GD, Brownsey RW, Allard MF. Energy metabolism in the hypertrophied heart. Heart Fail Rev. 2002;7(2):161-173.

57. Okere IC, et al. High-fat diet prevents cardiac hypertrophy and improves contractile function in the hypertensive dahl salt-sensitive rat. Clin Exp Pharmacol Physiol. 2005;32(10):825-831.

58. Taegtmeyer H, Ballal K. No low-fat diet for the failing heart? Circulation. 2006;114(20):2092-2093

59. Boudina S, et al. Mitochondrial energetics in the heart in obesity-related diabetes: direct evidence for increased uncoupled respiration and activation of uncoupling proteins. Diabetes. 2007; 56(10):2457-2466.

60. Laboissiere MC, Sturley SL, Raines RT. The essential function of protein-disulfide isomerase is to unscramble non-native disulfide bonds. J Biol Chem. 1995;270(47):28006-28009.

61. Wilkinson B, Gilbert HF. Protein disulfide isomerase. Biochim Biophys Acta. 2004;1699(1-2):35-44.

62. Burkart EM, et al. Nuclear receptors PPARbeta/ delta and PPARalpha direct distinct metabolic regulatory programs in the mouse heart. J Clin Invest.
2007;117(12):3930-3939.

63. Wang YX, et al. Regulation of muscle fiber type and running endurance by PPARdelta. PLoS Biol. 2004;2(10):e294.

64. Tontonoz P, Hu E, Graves RA, Budavari AI, Spiegelman BM. mPPAR gamma 2: tissue-specific regulator of an adipocyte enhancer. Genes Dev. 1994; 8(10):1224-1234.

65. Chawla A, Schwarz EJ, Dimaculangan DD, Lazar MA. Peroxisome proliferator-activated receptor (PPAR) gamma: adipose-predominant expression and induction early in adipocyte differentiation. Endocrinology. 1994;135(2):798-800.

66. Vikramadithyan RK, et al. Peroxisome proliferatoractivated receptor agonists modulate heart function in transgenic mice with lipotoxic cardiomyopathy. J Pharmacol Exp Ther. 2005;313(2):586-593.

67. Nesto RW, et al. Thiazolidinedione use, fluid retention, and congestive heart failure: a consensus statement from the American Heart Association and American Diabetes Association. Circulation. 2003;108(23):2941-2948.

68. Dormandy JA, et al. Secondary prevention of macrovascular events in patients with type 2 diabetes in the PROactive Study (PROspective pioglitAzone Clinical Trial In macroVascular Events): a randomised controlled trial. Lancet. 2005; 366(9493):1279-1289.

69. Staels B. Fluid retention mediated by renal PPARgamma. Cell Metab. 2005;2(2):77-78.

70. Marfella R, et al. Myocardial lipid accumulation in patients with pressure-overloaded heart and metabolic syndrome. J Lipid Res. 2009;50(11):2314-2323.

71. Taegtmeyer H, Wilson CR. Obesity and the risk of heart failure. NEngl J Med. 2002;347(23):1887-1889.

72. Akbar DH, Al-Gamdi AA, Hejazi NA. Poor lipid control in type- 2 diabetics with and without ischemic heart disease. Endocrine. 2003;21(3):217-220.

73. Poornima IG, Parikh P, Shannon RP. Diabetic cardiomyopathy: the search for a unifying hypothesis. Circ Res. 2006;98(5):596-605.

74. Yang J, et al. CD36 deficiency rescues lipotoxic cardiomyopathy. Circ Res. 2007;100(8):1208-1217.

75. Duncan JG, et al. Rescue of cardiomyopathy in peroxisome proliferator-activated receptor-alpha transgenic mice by deletion of lipoprotein lipase identifies sources of cardiac lipids and peroxisome proliferator-activated receptor-alpha activators. Circulation. 2010;121(3):426-435.

76. Folch J, Lees M, Sloane Stanley GH. A simple method for the isolation and purification of total lipides from animal tissues. J Biol Chem. 1957;226(1):497-509.

77. Lepage G, Roy CC. Direct transesterification of all classes of lipids in a one-step reaction. J Lipid Res. 1986;27(1):114-120.

78. Shen X, et al. Cardiac mitochondrial damage and biogenesis in a chronic model of type 1 diabetes. Am J Physiol Endocrinol Metab. 2004;287(5):E896-E905.

79. Zhang D, et al. Mitochondrial dysfunction due to long-chain Acyl-CoA dehydrogenase deficiency causes hepatic steatosis and hepatic insulin resistance. Proc Natl Acad Sci US A. 2007;104(43):17075-17080.

80. Devereux RB, et al. Echocardiographic assessment of left ventricular hypertrophy: comparison to necropsy findings. Am J Cardiol. 1986;57(6):450-458.

81. Seo T, et al. Lipoprotein lipase-mediated selective uptake from low density lipoprotein requires cell surface proteoglycans and is independent of scavenger receptor class B type 1. J Biol Chem. 2000; 275(39):30355-30362.

82. Boudina S, Abel ED. Diabetic cardiomyopathy revisited. Circulation. 2007;115(25):3213-3223.

83. Perry DK, Bielawska A, Hannun YA. Quantitative determination of ceramide using diglyceride kinase. Methods Enzymol. 2000;312:22-31.

84. Yu C, et al. Mechanism by which fatty acids inhibit insulin activation of insulin receptor substrate-1 (IRS-1)associated phosphatidylinositol 3-kinase activity in muscle. J Biol Chem. 2002;277(52):50230-50236

85. Laybutt DR, Schmitz-Peiffer C, Saha AK, Ruderman NB, Biden TJ, Kraegen EW. Muscle lipid accumulation and protein kinase $\mathrm{C}$ activation in the insulin-resistant chronically glucose-infused rat. Am J Physiol. 1999;277(6 pt 1):E1070-E1076.

86. Faiola B, et al. PPAR alpha, more than PPAR delta, mediates the hepatic and skeletal muscle alterations induced by the PPAR agonist GW0742. Toxicol Sci. 2008;105(2):384-394. 